\title{
Anthropometric baseD Estimation of adiPoSity - The ADEPS Project
}

\author{
Willem DE KEYZER ${ }^{* 1}$, Frank DERUYCK ${ }^{2}$, Benjamin VAN DER SMISSEN ${ }^{3}$, Simona VASILE ${ }^{4}$, \\ Joris COOLS ${ }^{4}$, Alexandra DE RAEVE ${ }^{4}$, Stefaan DE HENAUW ${ }^{5,1}$, Peter VAN RANSBEECK ${ }^{3}$ \\ ${ }^{1}$ Bio- and food sciences, University College Ghent, Ghent, Belgium; \\ ${ }^{2}$ Exact sciences, University College Ghent, Ghent, Belgium; \\ ${ }^{3}$ Mechatronics, University College Ghent, Ghent, Belgium; \\ ${ }^{4}$ Fashion, textile and wood technology, University College Ghent, Ghent, Belgium; \\ ${ }^{5}$ Public health, Ghent University, Ghent, Belgium
}

DOI: 10.15221/15.085 http://dx.doi.org/10.15221/15.085

\begin{abstract}
Worldwide, the prevalence of obesity has increased dramatically. Obesity is a condition associated with an increased amount of adipose tissue in the body and is linked to increased morbidity and mortality. In clinical practice and research, determination of body fat percentage (\%BF) is not always possible due to limitations in available resources (time, equipment, budget, etc.). Therefore, weight indexes like the body mass index (BMl; body weight $(\mathrm{kg}) /$ body height ${ }^{2}(\mathrm{~m})$ ) offer a major advantage because they are quick and inexpensive to use. Although the BMI is extensively used, it does not take into account fat or muscle distribution in the body and is unable to differentiate adipose tissue from lean body mass. Hence, it has been suggested that future research in body composition measurement should focus more on body shape and volume rather than body mass. With the advent of $3 \mathrm{D}$ body scanning technology, it is possible to obtain accurate and reliable anthropometric measures of an individual within a few minutes. Also, 3D body scans provide information on an individual's body volume and body shape. From this data, \%BF can be calculated using a two component model of the human body based on known densities of fat and fat-free mass. In addition, a 3D digital model of the body allows for visualization of regional fat deposition and division of the total body into segments for more detailed data analysis compared to total body measurements. The ADEPS project builds on experience with 3D body scanning gained during the SMARTFIT project and is looking to merge areas of expertise in medicine, health care and technology. The principal aim of the ADEPS project is to examine the extent to which \%BF can be predicted using anthropometric measurements obtained from $3 \mathrm{D}$ body scans using a structured white light full body scanner. A comprehensive dataset of anthropometric measurements obtained by 3D body scanning is available within the research unit. From these data, samples of candidate anthropometrical measurements will be selected using a Design of Experiments approach. Regression analysis on sequentially selected datasets will yield anthropometric predictors which will be used to create a predictive model for \%BF as calculated from total body volume. This model will then be validated by comparing the anthropometric-based \%BF predictions with \%BF obtained from the Bod Pod $\Omega$ air-displacement plethysmography system (reference method and gold standard for total body volume measurement). Finally, the regression equation will be converted into a nomogram for routine practical use in healthcare and research practice. The present article describes the research project and its methods and reports on the progress and intermediate results of the ADEPS project.
\end{abstract}

Keywords: 3D body scanning, air-displacement plethysmography, body composition, obesity, body fat, body shape, body volume

\section{Introduction}

The prevalence of obesity has increased dramatically worldwide (Fig. 1). According to the World Health Organization (WHO), the number of people struggling with obesity today has doubled since 1980. Obesity is a condition in which the amount of fat tissue is increased resulting in adverse effects on health and is associated with increased morbidity and mortality [1]. This state of fat accumulation (adiposity, Latin: adeps), is linked to increased risks of type 2 diabetes, cardiovascular disease, fatty liver, sleep apnea syndrome and certain types of cancer [2] and a decreased life expectancy [1,3].

*dr. Willem De Keyzer, willem.dekeyzer@hogent.be; +32 924323 72; fnt.hogent.be 


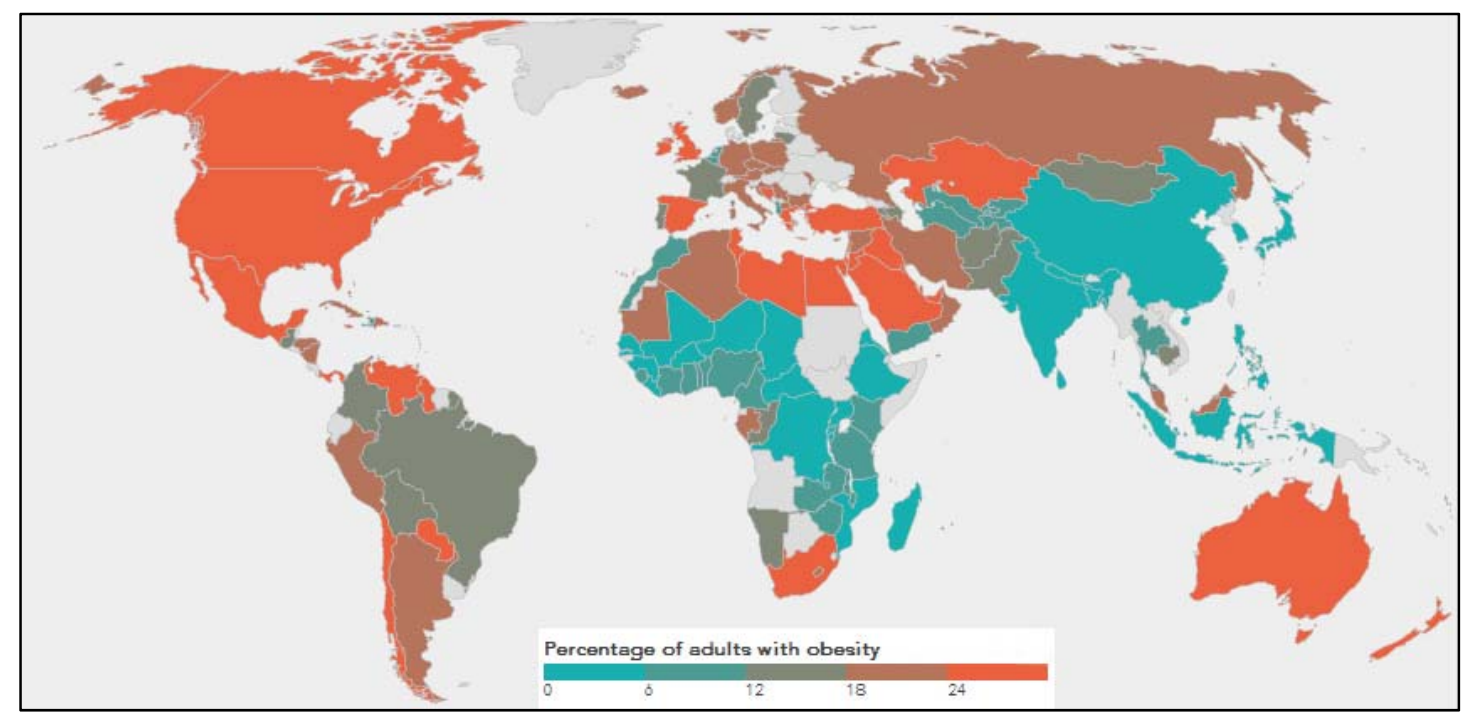

Fig.1. World map of adult obesity prevalence (http://www.worldobesity.org).

Obesity-related complications rise from the excess fat tissue and its localization apart from the excess weight $[4,5]$. This also explains the interest of researchers and clinicians to assess the amount of total body fat percentage (\%BF) [6]. \%BF can be measured using various techniques, ranging from skinfold thickness measurements up to the use of magnetic resonance imaging (MRI) [7]. Other commonly used methods for determining \%BF involve bioelectrical impedance analysis (BIA) and dual-energy X-ray absorptiometry (DEXA). More accurate and reproducible methods include measurements based on body density such as underwater weighing and Air Displacement Plethysmopgraphy (ADP) [7-9].

However, determination of \%BF is not always possible because of costs, available time or limited access to the necessary measuring devices. Under these circumstances the body mass index (BMI) is often used as a proxy measure for the assessment of the degree of adiposity. Nevertheless, although the BMI is quick and easy to calculate, this parameter has some major shortcomings. For instance, the BMI is not an accurate measure for the amount of body fat and insensitive to sexual dimorphism and changes in body composition during aging [10-13]. Thus, while BMI is a good predictor of weight-related morbidity on the population level, it is not as effective in individual cases. Body weight is influenced by more than fat tissue, including high muscle mass, and the relationship between $\mathrm{BMI}$ and health can vary with ethnicity.

In the scientific literature several multiple linear regression equations are available in which age and gender, in addition to BMI, are put into a predictive model in order to predict \%BF [3, 11, 14-21]. Analogous to these equations describing a relationship between \%BF and $\mathrm{BMI}$, additional anthropometric parameters are possibly able to predict \%BF also. The current published regression equations produce $\mathrm{R}^{2}$-values ranging from 0.38 to 0.79 so a considerable part of the variance in $B F \%$ $(62-21 \%)$ remains unexplained by these models. Traditionally, anthropometric data are analyzed for their potential to serve as predictors variables for \%BF. However, the number of anthropometric parameters that are usually studied are rather limited and their choice often results from what researchers consider to be reasonable predictor variables. In the ADEPS project we are able to screen more than 150 anthropometric predictors obtained from 3D body scans using systematic experimental statistical approaches in order to increase predictive capacity of \%BF estimation models.

\section{Methods}

\subsection{Study design}

The ADEPS project aims to determine total body volume and specific areas of fat deposition using 3D body scanning. From a set of bodyscans, a post-processing procedure will be developed to determine total body volume so these volumes can be used to calculate \%BF. In a next phase, it will be assessed which anthropometric measurements provided by the body scan are useful predictors for \%BF resulting in a predictive model. Finally, this model will be validated using a reference method for \%BF determination and resulting predictive equations will be converted into a nomogram for convenient assessment of \%BF from simple and manual measurable anthropometrics. 


\subsection{Statistical design}

Several anthropometric parameters influence \%BF so in order to generate a reliable model, correct sampling is of utmost importance and therefore the Design of Experiments (DOE) approach [22] is used ensuring that:

i. Sampling occurs over a sufficiently broad range of all potential variables and categories (stratified sampling);

ii. $\quad$ There is no or only little correlation between the input parameter settings;

iii. A minimal sample size is obtained for estimating model coefficients with maximal precision;

iv. The "Goal - Model - Design" criterion is fulfilled i.e. by statistical DOE result analysis it must be possible to yield the target model linked to the study objective: in this work the goal is to construct an optimal model for describing \%BF so a full quadratic (response surface) model is required for which an appropriate DOE must be selected; in the screening stage a Definitive Screening Design will be necessary.

The final model buildup is sequential and occurs in three consecutive phases:

1. Screening stage: identification of the main $\left(1^{\text {st }}\right.$ order $)$ and quadratic effects; a Definitive Screening Design is used [23];

2. Optimization stage: based on the screening results a DOE will be chosen to construct a candidate set of potential predictive models;

3. Validation stage: the optimization stage DOE is used to yield a model validation sample. The final optimal model is the one with the highest prediction power.

The \%BF regression models are constructed by statistical analysis of the DOE results; this is an iterative process as presented in figure 2 .

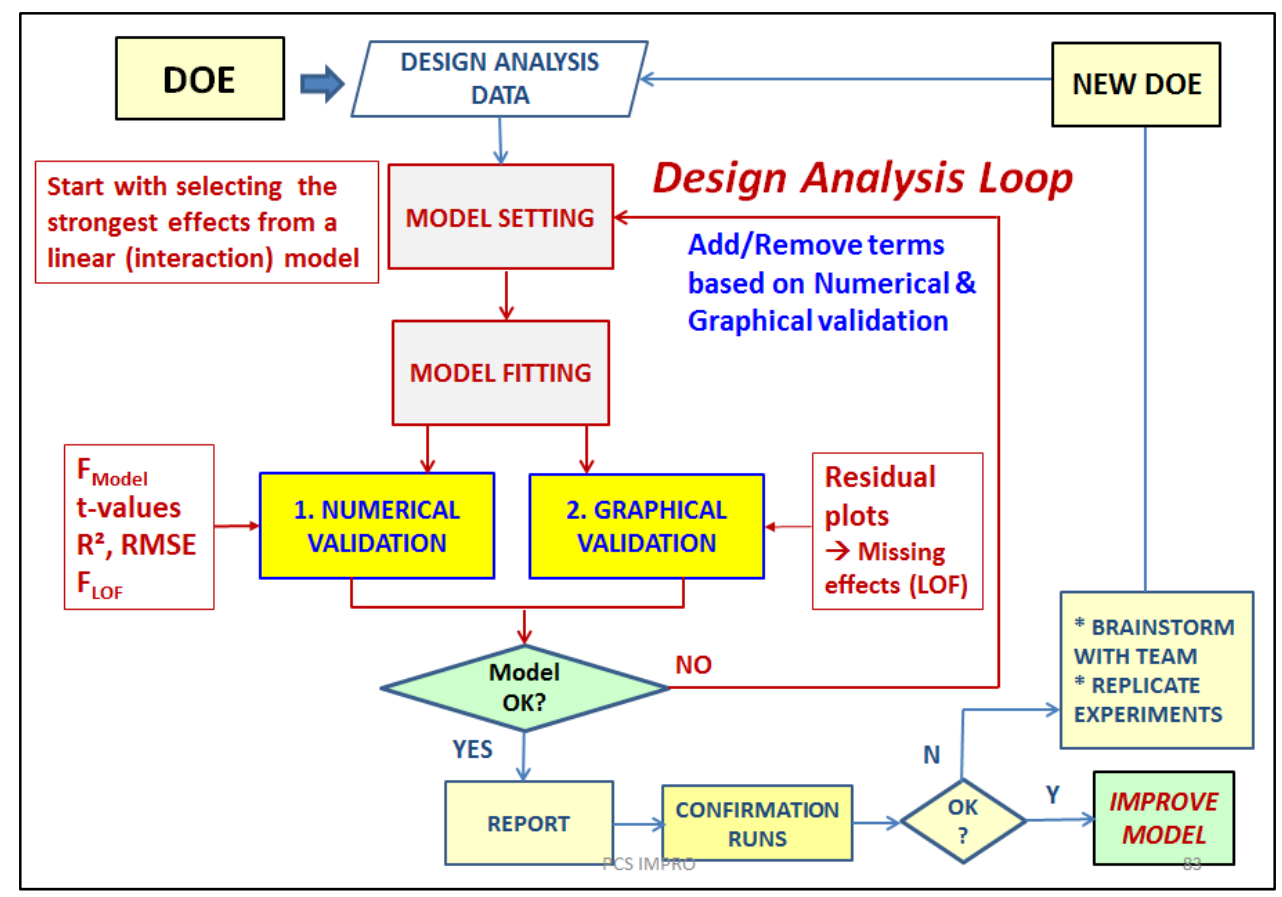

Fig. 2 . Statistical design analysis flowchart of the ADEPS project.

\subsection{Target population}

The research focuses on adult participants of both sexes (18-65 years) living in Belgium. Special attention will be given to the inclusion of participants from different weight categories. The upper limit for body weight is set to $250 \mathrm{~kg}$.

\subsection{D Body scanner}

During the Smartfit campaign approximately 2500 persons between 3 and 70 years $(0,02 \%$ of the Belgian population) were arbitrary chosen and measured. We made use of two 3D scanning booths: a Telmat Symcad and a TC ${ }^{2}$ NX-16 body scanner. Both systems use structured white light technology and have a 3D-point accuracy of less than $1 \mathrm{~mm}$. The circumferential accuracy for measurement 
extraction is less than $3 \mathrm{~mm}$. The NX-16 scanner has 32 camera's, 8 in each angle. The Symcad scanner has 4 camera's, 2 in front and 2 in the rear. The scanning volume of both scanners is comparable. The acquisition time for the NX-16 is 7 to 8 seconds, for the Symcad only 1,5 second, while the measurement extraction time is comparable. With each system, the maximum capacity is about 25 measurements per hour.

All participants were measured according to the same measure protocol: standing posture with the head in Frankfort plane, the legs slightly apart, the arms bent while making a fist and breathing normally. 180 measurements were extracted by the software according the standard ISO 8559 . Total body height, weight, head girth and waist height while sitting, were taken manually. $75 \%$ of these measurements are stored for future applications, 45 variables were used for the calculation of 25 average body measurements in 7 different body measurement tables, each split into 3 tables: garments for full body, upper body and lower body.

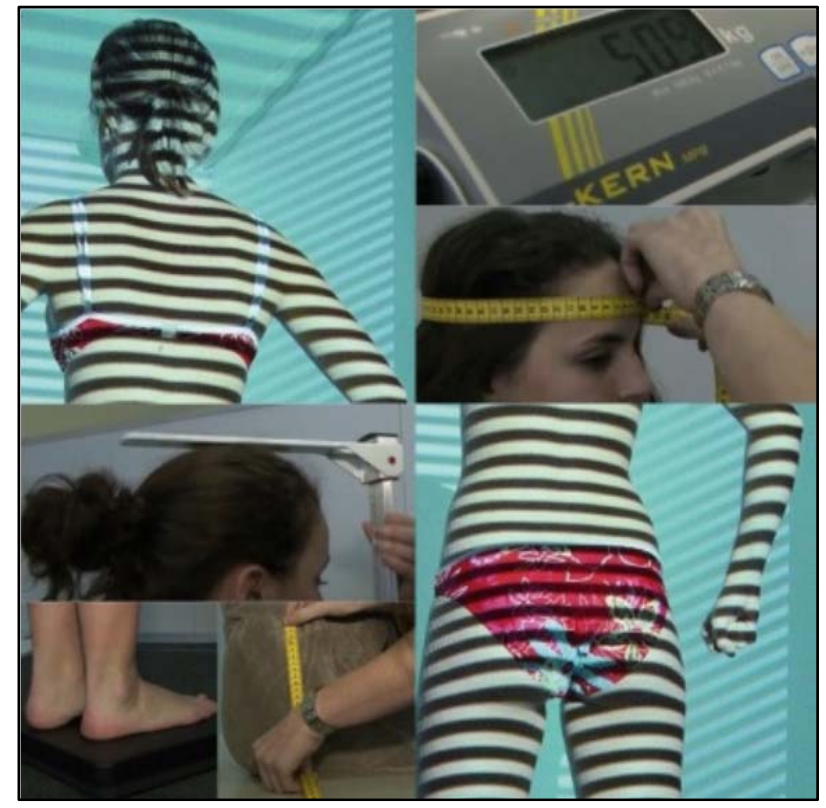

Fig. 3. Measurement protocol.

\subsection{Whole-body air displacement plethysmography}

Whole-body air displacement plethysmography (ADP) is a validated densitometric method to indirectly measure total \%BF $[8,24]$. The underlying principle for ADP is identical as for the gold-standard method involving underwater weighing (based on Archimedes' principle). However, the densitometric method is based on air displacement rather than on water immersion. Air-displacement plethysmography offers several advantages over established reference methods, including a quick, comfortable, automated, non-invasive, and safe measurement process, and accommodation of various subject types (e.g., children, obese, elderly, and disabled persons) [8]. With ADP, the volume of a person is measured indirectly by determining the volume of air it displaces inside an enclosed capsule (Fig. 4). In practice, human body volume is measured when a person sits inside an airtight chamber displacing a volume of air equal to his or her body volume. Body volume is then calculated indirectly by subtracting the volume of air remaining inside the chamber when the subject is inside from the volume of air in the chamber when it is empty. The volume of air inside the chamber is calculated by slightly changing the size of the chamber using an oscillating membrane and applying relevant physical gas laws to determine the total volume from the changing air pressure within the chamber as its size is altered. The densitometric method assumes that the body consists of two components, a fat mass, in which all chemical fat is located, and the fat-free mass (FFM), which consists of bones, muscles, water, and organs. The density of the fat mass is $0.900 \mathrm{~kg} / \mathrm{l}$ and, from carcass analysis data, the density of the FFM can be calculated as $1.100 \mathrm{~kg} / \mathrm{l}$, depending on the relative amount of minerals, protein, and water in the FFM [6]. The density of the total body depends on the ratio of fat mass to FFM. From total body volume and body weight, the density of the body and $\% \mathrm{BF}$ can be calculated. 


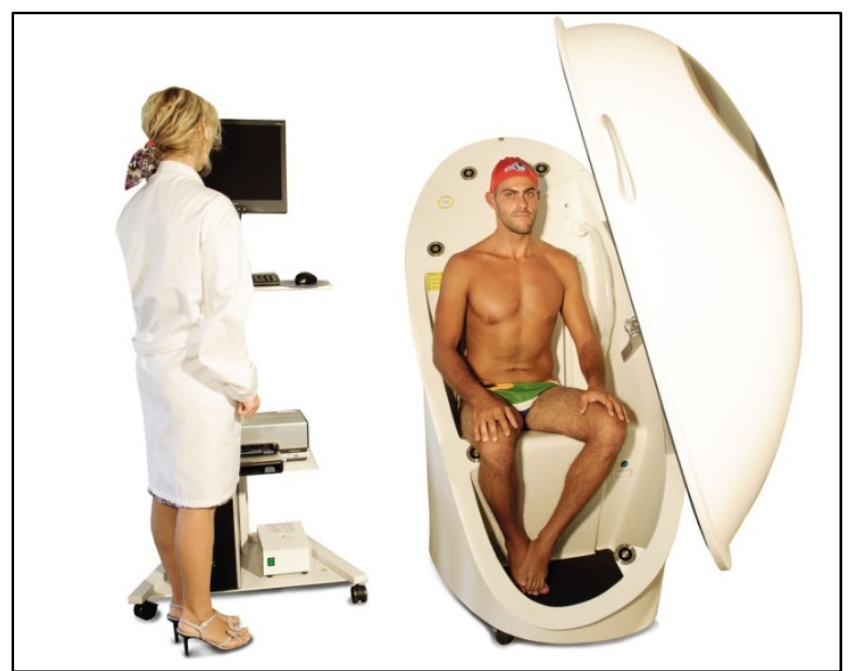

Fig.4. Body composition measurement in adults with air displacement plethysmography technique by COSMED (http://www.cosmed.com).

\section{Intermediate project results}

\subsection{Selection of candidate predictors}

Anthropometrics from the SMARTFIT project [25] were evaluated for their strongest correlation with dependent variables in two stages using BMI and total body volume as outcome variables.

Firstly, to generate a first candidate sample (Definitive Screening Design), correlations of anthropometric measures with BMI were evaluated (Definitive Screening Design). This approach was chosen since our SMARTFIT data did not contain measures of adiposity. In females, four anthropometric measures (waist girth, body height, neck girth, calf girth) and two calculated measures (body weight/waist girth, bust girth/waist girth) were retained because of their strong correlation with BMI $\left(r^{2}=0,90 ; R M S E=1,10\right)$. In males, also four anthropometric measures (waist girth, body height, neck girth, thigh girth) and two calculated measures (body weight/waist girth, bust girth/hip girth) were retained $\left(r^{2}=0,89\right.$, RMSE $\left.=1,08\right)$. Using tertile cut-off points of the continuous measurements, three categories were defined (e.g. waist circumference in females; cat1: <67 cm, cat2: $67-79 \mathrm{~cm}$, cat3: > $79 \mathrm{~cm}$ ). Next, body scans from the SMARTFIT database were selected covering all tertile categories of the retained measurements to maximize diversity in body shape ( $n=17$ per gender category). All selected body scans were then post-processed for determination of total body volume (see 3.2 post processing).

Secondly, correlations of anthropometrics with total body volume obtained using post-processing of body scans were evaluated. The strongest model in females included waist girth, body height, thigh girth and wrist girth $\left(r^{2}=0,96\right.$, RMSE $=1,46$, Fig. 5). In males the model included waist girth, body height, thigh girth and upper arm girth $\left(r^{2}=0,98\right.$, RMSE $=1,58$, Fig.6).

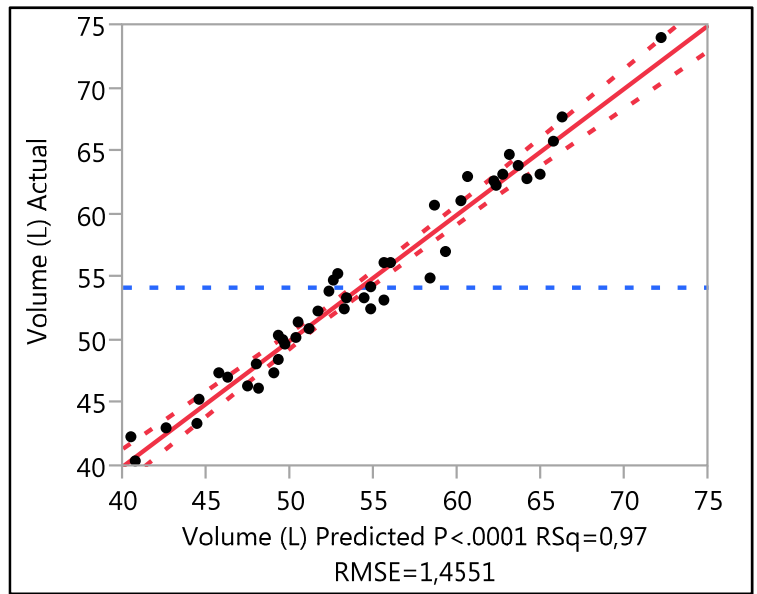

Fig. 5. Actual by predicted plot of total body volume in females $(n=46)$.

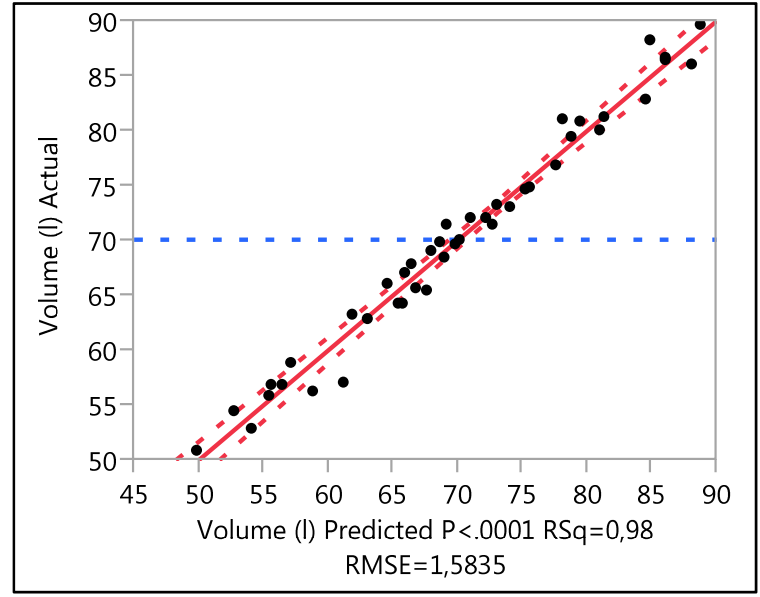

Fig. 6. Actual by predicted plot of total body volume in males $(n=43)$. 


\subsection{Post processing}

The SymCad scans were processed using Artec studio 9 software (Artec Group, Luxembourg) according to the following procedure (Fig. 7). First, the fusion operation was applied and resulted in a smoothed watertight model. Then, due to inconsistencies of head, hands and feet scans, it was decided to remove these parts at specific regions defined by ISO 8559 (1989) by the eraser operation. After that, gaps - which were caused by the latter operation - were closed by the holes operation. Finally, the volume of the 3D body without head, hands and feet $\left(\mathrm{V}_{-\mathrm{HHF}}\right)$ was calculated by the software.

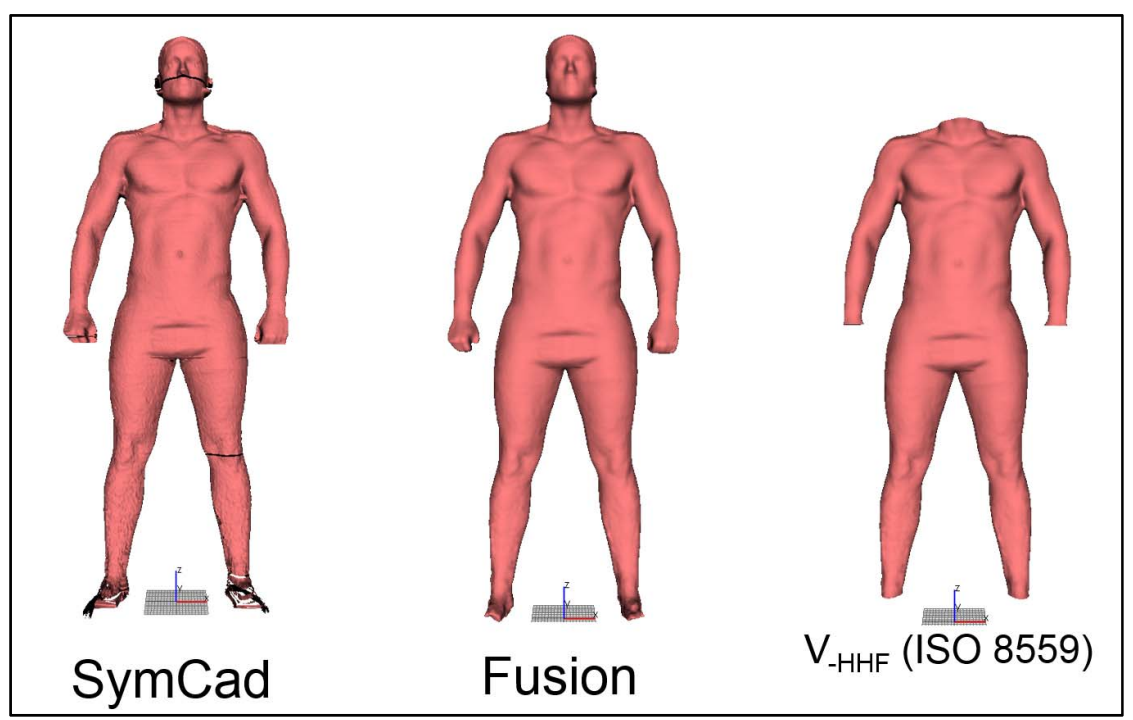

Fig. 7. Post processing steps of body scans ( $V_{-H H F}$ : total body volume minus head, hands and feet).

\section{Conclusions and future steps}

The ADEPS project aims to identify key anthropometric data that enables us to estimate \%BF from readily available and manually measurable body dimensions. The approach that is used is innovative in this sense that, 1) a large dataset of $3 D$ body scans representing the adult Belgian population is used for this objective, 2) rigor statistical methods are used to identify potential predictor variables for \%BF, and 3) apart from model validation using existing data, our final models will be validated for which a new sample will be measured with both a 3D body scanner and a gold standard method for total body volume measurements.

So far the project has shown that post processing procedures of body scans are able to estimate total body volume. This procedure has shown to be challenging, especially if head, hands and feet are to be included. On average, the body scan-based volumes underestimate about $8 \%$ of total body volume. Nevertheless, our analysis shows that when excluding these body parts, more than $96 \%$ of variation in body volume can be predicted using a selection of anthropometric measurements.

In the next phase of the ADEPS project, a new sample will be measured using the abovementioned instruments. This will enable us to validate our prediction models with new data points and allows us to calibrate \%BF predictions of the SMARTFIT sample.

\section{References}

[1] A. Berrington de Gonzalez, P. Hartge, J. R. Cerhan, A. J. Flint, L. Hannan, R. J. Maclnnis, et al., "Body-mass index and mortality among 1.46 million white adults," $N$ Engl $J$ Med, vol. 363, pp. 2211-9, Dec 22010.

[2] D. W. Haslam and W. P. James, "Obesity," Lancet, vol. 366, pp. 1197-209, Oct 12005.

[3] B. L. Heitmann, "Evaluation of body fat estimated from body mass index, skinfolds and impedance. A comparative study," Eur J Clin Nutr, vol. 44, pp. 831-7, Nov 1990.

[4] P. Deurenberg, M. Yap, and W. A. van Staveren, "Body mass index and percent body fat: a meta analysis among different ethnic groups," Int J Obes Relat Metab Disord, vol. 22, pp. 1164-71, Dec 1998. 
[5] WHO, "World Health Organization - Programme of Nutrition, Family and Reproductive Health. Obesity : preventing and managing the global epidemic : report of a WHO Consultation on Obesity, Geneva, 3-5 June 1997," Geneva WHO/NUT/NCD/98.1, 1998.

[6] P. Deurenberg, A. Andreoli, P. Borg, K. Kukkonen-Harjula, A. de Lorenzo, W. D. V. Lichtenbelt, et al., "The validity of predicted body fat percentage from body mass index and from impedance in samples of five European populations," European Journal of Clinical Nutrition, vol. 55, pp. 973979, Nov 2001.

[7] S. K. Das, "Body composition measurement in severe obesity," Curr Opin Clin Nutr Metab Care, vol. 8, pp. 602-6, Nov 2005.

[8] D. A. Fields, M. I. Goran, and M. A. McCrory, "Body-composition assessment via airdisplacement plethysmography in adults and children: a review," Am J Clin Nutr, vol. 75, pp. 45367, Mar 2002.

[9] S. R. Ginde, A. Geliebter, F. Rubiano, A. M. Silva, J. Wang, S. Heshka, et al., "Air displacement plethysmography: validation in overweight and obese subjects," Obes Res, vol. 13, pp. 1232-7, Jul 2005.

[10] D. Gallagher, M. Visser, D. Sepulveda, R. N. Pierson, T. Harris, and S. B. Heymsfield, "How useful is body mass index for comparison of body fatness across age, sex, and ethnic groups?," Am J Epidemiol, vol. 143, pp. 228-39, Feb 11996.

[11] A. S. Jackson, P. R. Stanforth, J. Gagnon, T. Rankinen, A. S. Leon, D. C. Rao, et al., "The effect of sex, age and race on estimating percentage body fat from body mass index: The Heritage Family Study," Int J Obes Relat Metab Disord, vol. 26, pp. 789-96, Jun 2002.

[12] A. M. Prentice and S. A. Jebb, "Beyond body mass index," Obes Rev, vol. 2, pp. 141-7, Aug 2001.

[13] M. B. Snijder, R. M. van Dam, M. Visser, and J. C. Seidell, "What aspects of body fat are particularly hazardous and how do we measure them?," Int $J$ Epidemiol, vol. 35, pp. 83-92, Feb 2006.

[14] J. S. Garrow and J. Webster, "Quetelet's index (W/H2) as a measure of fatness," Int J Obes, vol. 9, pp. 147-53, 1985.

[15] N. G. Norgan and A. Ferro-Luzzi, "Weight-height indices as estimators of fatness in men," Hum Nutr Clin Nutr, vol. 36, pp. 363-72, 1982.

[16] J. Womersley, "A comparison of the skinfold method with extent of 'overweight' and various weight-height relationships in the assessment of obesity," Br J Nutr, vol. 38, pp. 271-84, Sep 1977.

[17] P. Deurenberg, J. A. Weststrate, and J. C. Seidell, "Body mass index as a measure of body fatness: age- and sex-specific prediction formulas," Br J Nutr, vol. 65, pp. 105-14, Mar 1991.

[18] M. Visser, E. van den Heuvel, and P. Deurenberg, "Prediction equations for the estimation of body composition in the elderly using anthropometric data," $\mathrm{Br} J$ Nutr, vol. 71, pp. 823-33, Jun 1994.

[19] T. L. Dupler and H. Tolson, "Body composition prediction equations for elderly men," Journals of Gerontology Series a-Biological Sciences and Medical Sciences, vol. 55, pp. M180-M184, Mar 2000.

[20] J. Gomez-Ambrosi, C. Silva, V. Catalan, A. Rodriguez, J. C. Galofre, J. Escalada, et al., "Clinical usefulness of a new equation for estimating body fat," Diabetes Care, vol. 35, pp. 383-8, Feb 2012.

[21] D. Martarelli, B. Martarelli, and P. Pompei, "Body composition obtained from the body mass index: an Italian study," Eur J Nutr, vol. 47, pp. 409-16, Dec 2008.

[22] R. H. Myers, D. C. Montgomery, and C. M. Anderson-Cook, Response surface methodology: process and product optimization using designed experiments vol. 705: John Wiley \& Sons, 2009.

[23] B. Jones and C. J. Nachtsheim, "A class of three-level designs for definitive screening in the presence of second-order effects," Journal of Quality Technology, vol. 43, pp. 1-15, 2011.

[24] M. A. McCrory, T. D. Gomez, E. M. Bernauer, and P. A. Mole, "Evaluation of a new air displacement plethysmograph for measuring human body composition," Med Sci Sports Exerc, vol. 27, pp. 1686-91, Dec 1995.

[25] J. Cools, A. De Raeve, P. Van Ransbeeck, S. Vasile, B. Van Der Smissen, and M. Vermeulen, "The Use of 3D Anthropometric Data for Morphotype Analysis to Improve Fit and Grading Techniques - The Results," presented at the 5th International Conference on 3D Body Scanning Technologies, Lugano, Switzerland, 2014. 